\title{
Development of swing phase relearning device
}

\author{
I. Cikajlo, Z. Matjačić \& T. Bajd \\ University of Ljubljana, Faculty of Electrical Engineeers, Trǎaška 25, \\ 1000 Ljubljana, Slovenia \\ 'Institute for Rehabilitation, Linhartova 51, 1000 Ljubljana, Slovenia
}

\begin{abstract}
The aim of the paper is to present development and use of the functional electrical stimulation (FES) gait reeducation system in swing phase rehabilitation of the incomplete spinal cord injured persons. The proposed system consists of four accelerometers, gyro placed at the shank of the paretic leg and two goniometers, placed in knee and ankle joint. The data assessed from sensors present input into mathematical algorithms applied for swing quality estimation that equals to a single numerical value. This value is divided in three level output to determine the state of cognitive feedback (CF). Each level defines the swing quality in terms of good, sufficient and poor and is provided to the patient as an auditory signal. A single channel peroneal FES was applied to provide the needed assistance to the
\end{abstract}




\section{6}

Simulations in Biomedicine $V$

patient. The assistance was provided only to the level that enabled the patient to perform a swing. The automatic FES control was handled by personal computer. Regarding to the quality of the performed swings the intensity of FES was increased (pre-set number of consecutive poor swings) or decreased (pre-set number of consecutive good swings), forcing the patient to voluntary contribute to swing improvement.

Developed system was applied and tested with a single incomplete spinal cord injured (SCI) patient with C4-5 lesion during walking on the treadmill. The results have shown that involving the patient in the rehabilitation process with the automatic FES control, providing only the minimum needed assistance, can improve the training of walking. Hopefully the FES assistance would diminish after the training period. Therefore, the use of FES, multisensor and cognitive feedback might be efficient rehabilitative approach in early stage of rehabilitation.

\section{Introduction}

These days the safety in traffic and vehicles has been improved. Consequently the number of complete SCI patients has reduced and more incomplete cases are arriving in spinal units. Most of the incomplete SCI patients are candidates for use of the FES rehabilitation approach soon after the accident [1]. Quite a large number of FES patient have shown deficiencies during walking, especially disturbances in swing phase. The idea of qualitative measurement and swing estimation have brought to opportunity to develop a system which will support the conventional FES.

During decades researches have developed several systems coupling FES and artificial sensors. Petrofsky [2] used a closed loop system to control the electrical stimulation in complete paralysed individuals. Phillips et al [3] built a FES ortho- 
sis and showed the use of auditory feedback during walking in spinal cord injury. He examined different types of auditory feedback system with respect to the various walking velocities. The walking quality has improved by using any kind of auditory feedback, but there was no significant difference when employing more than one type. Mulder's [4] study about human perception emphasised the concept of the accepted sensory information. FES assisted walking in incomplete SCI using sensory feedback information was presented by Erzin [5]. Compact wearable system consisting of goniometers and foot-switches was used to determine the appropriateness of the swing phase on the basis of the basogram.

The aim of the proposed FES gait re-education system is to estimate the quality of the swing phase of walking and involve patients in the process of repeatable training. As the actuation system simple one-channel FES is proposed for use with paretic persons. Electrical stimulation of spinal neural circuits, rather than direct activation of motoneurons, is simplifying generation of complex motor behaviours. In order to accomplish the swing phase of walking, the peroneal stimulation should be considered, along with the ankle plantar flexors stimulation [6]. In incomplete SCI patients one leg is usually considerably more affected than the other. FES is therefore usually applied only unilaterally. We propose the automatic FES triggering and intensity control to achieve repeatability. This relieves the patient of FES control and enables to concentrate on cognitive feedback (CF) and his walking. When his walking becomes better, the FES intensity is automatically decreased and when poorer increased but only to the level of needed assistance. This approach force the patient to take an active part in rehabilitation. 


\section{Methods}

The proposed approach is based on two control loops (Fig. 1), one of them handles the automatic control of the FES and the other involves the patient into the rehabilitation process. The latter, the more important loop, handles the processed sensory information of estimated swing quality [7] and provide it as a auditory feedback. The swing quality estimation is based on multisensor integration. Assessed data from knee goniometer, accelerometers and gyroscope are used to determine the numerical value [7], which represents the consistency with the desired reference swing. The reference swing could be chosen either from the less affected lower extremity of the SCI patient or the healthy extremity. The numerical value is provided to the patient as auditory feedback in three different levels. The levels are presented as three various frequencies. The low frequency is adequate to the poor swing, higher frequency to the good swing and the high frequency sound represents an excellently performed swing phase. Described feedback information is simple enough to be perceived during walking and enables the patient to voluntarily improve the swing of his affected lower extremity.

The FES control loop is based on the assessed sensory information and the swing quality. The sensors provide the necessary information to determine the moment of triggering of the electrical stimulation. Since we use the single channel peroneal nerve stimulation to provoke the flexion response, i.e., simultaneous hip and knee flexion and dorsiflexion, we have to make sure that the moment of triggering will take place before the initial swing phase. We defined the appropriate moment of FES triggering using the information from goniometer, placed at the knee joint. When the knee flexion occurs, the heel-off phase takes place and from numerous trials this appeared to be the best triggering moment of the peroneal stimulation. 


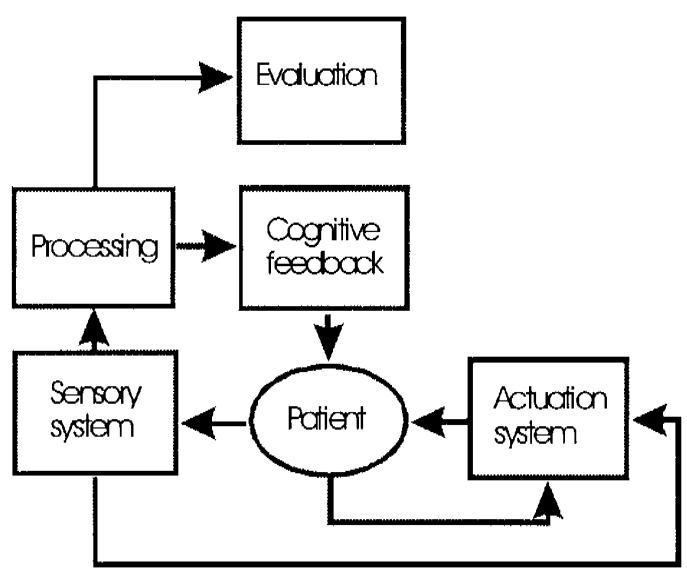

Figure 1: The complete idea of neurorehabilitation sheme. The patient is involved in the rehabilitation process. The sensory system is used to activate the actuation system and for cognitive feedback, which is provided to the patient. Therefore the patient is aware of his walking and is able of voluntary reaction.

The stimulation intensity is pre-set by the physiotherapist according to the patient deficits. During walking the patient swings are being estimated and when pre-set number of good/poor consecutive swings have been performed then the stimulation intensity is decreased/increased.

\subsection{Instrumentation}

The measurements were carried out on the treadmill in the rehabilitation centre. Most of the processing was managed by personal computer (PC, Pentium III 500 $\mathrm{MHz}$ ), based on Windows platform. The supervisor software was programmed in Matlab/Simulink and C++. Signals were assessed by a multi-sensor device [7], 
two pair of single crossaxial accelerometers, a gyroscope and goniometers in ankle and knee joint. The algorithm estimated the swing quality [7]. The user-friendly software provided quite a few options to set the required knee angle and consequently the moment of triggering, the required knee flexion for good swing phase, the number of swings required for automatic stimulation amplitude adjustment and the parameters of the FES. We chose the clinically very well known single channel surface peroneal nerve stimulation with the following parameters, stimulation frequency $20 \mathrm{~Hz}$, pulse width $200 \mathrm{~ms}$, current $35 \mathrm{~mA}$.

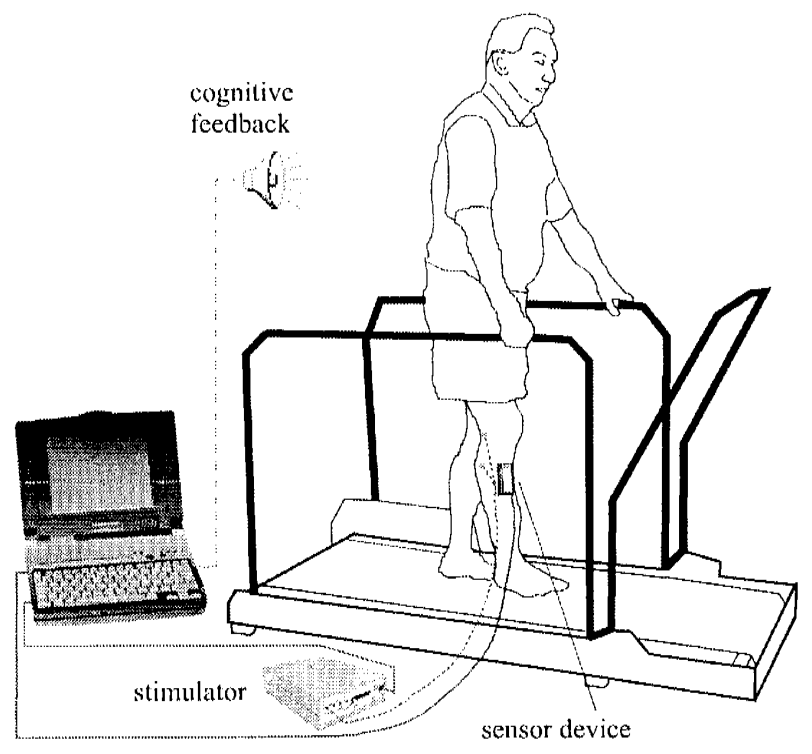

Figure 2: The FES swing re-education set-up. Sensor assesses data from the affected extremity and send it to the process unit (PC). The process unit controls the electrical stimulator and provides the cognitive feedback. 


\section{Results}

The testing of the developed swing phase learning was performed on one incomplete SCI patient $(175 \mathrm{~cm}, 84 \mathrm{~kg}, 30 \mathrm{y}$, male) with C4-C5 lesion (4 months after the accident). He was not able to perform a swing without FES and was a regular MicroFES (The Jozef Stefan Institute) user for 1 month. While he was using the MicroFES stimulator, his walking was considered satisfactory. Therefore we measured his walking on the treadmill (speed $0.5 \mathrm{~m} / \mathrm{s}$ ) and considered it as a reference. But the goal is to achieve the same swing without or with minimal FES assistance, using only the CF and the patient's effort. In order to minimize the assistance we used a computer controlled electrical stimulator to replace the MicroFES on his right leg. The testing procedure we prescribed demanded from patient to concentrate on CF and try to voluntarily perform a swing. Of course the computer controlled stimulator provided the FES assistance, but only to the minimum needed level. The physiotherapist pre-set the stimulation parameters, the required knee flexion $\left(20^{\circ}\right)$ swing phase and the required number (5) of good/poor consecutive swings for the automatic change of the stimulation intensity $(10 \%)$. The intensity was also limited to prevent too much FES assistance.

The figure 3 shows the results taken during measurements. The upper diagram presents the presence and the intensity $(\mathrm{mA})$ of the FES. In the beginning, when the swing phase did not match the desired reference swing, the intensity of the FES remained at the pre-set value. This intensity could not satisfy the needed assistance, so the patient voluntary effort was necessary. Later when he catch up with the walking, the swing quality improved and consequently the FES assistance was decreased. By his effort and with the FES assistance his walking became symmetrical. When his concentration declined the stimulator increased the FES assisstance. 

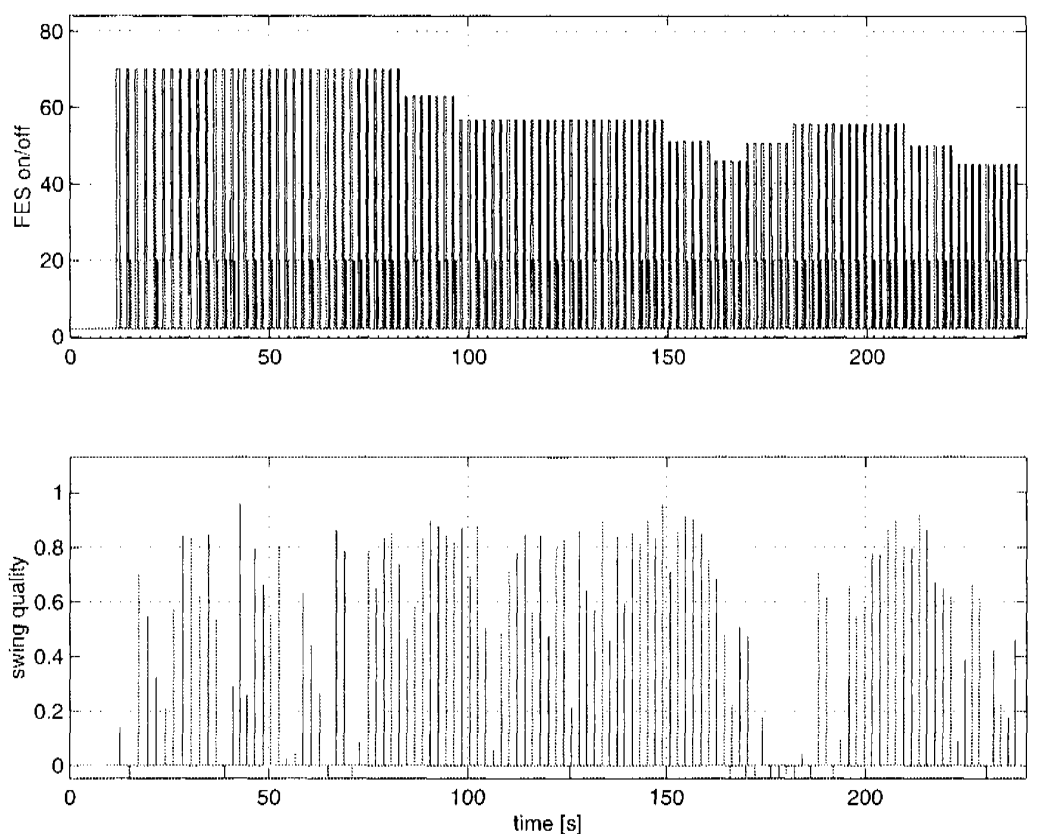

Figure 3: The FES assisted voluntary walking on the tredmill. If the patient's voluntary walking had become better, symmetrical, the FES assistance ( $\mathrm{mA}$ ) was decreased (upper diagram). The lower diagram presents the estimated swing quality in every step cycle $(0-$ poor, 1 - good $)$.

In the lower diagram a swing quality after every step is presented ( 0 - poor, 1 good). In a case of insufficient knee flexion the output was -1, but the CF was the same as would has been marked as poor. The training process lasted $4 \mathrm{~min}$., then a short break was needed because of the FES [6] in spite of the patient indefatigability. 


\section{Discussion}

One of our first approach in FES gait relearning suggested a manual triggering by the patient itself [8] or by the psychotherapist [9]. The first approach turned out as time-consuming so the patient put a lot of effort on the triggering. It is still very good, but deficient in training process. The second approach was very efficient letting the patient to concentrate on the $\mathrm{CF}$. As he was notified of his swing quality he was able to voluntarily change his walking pattern. The FES only gave him a necessary assistance. But to increase the repeatability of instant of triggering and duration of the FES we replaced the manual triggering by a computer controlled stimulator. When the estimated swing quality became good, the stimulator decreased the intensity and when poor increased it to the level of necessary assistance. This approach forced the patient to voluntarily move his lower extremity as much as he can. In Results we have shown the effectiveness of the proposed approach. The repeatability of the proposed automatic FES control could be efficient and perhaps it is a need during training process in the early phase of rehabilitation. Therefore the rehabilitation process could be shortened as well and we do expect that after a month of FES swing re-education the FES assistance might dwindle away due to the significant walking improvement.

\section{Acknowledgements}

The authors acknowledge the financial support of the Republic of Slovenia Ministry of Education, Science and Sport. Special thanks for assistance go to Pavla Obreza, PT and Janez Šega, dipl.ing. 


\section{References}

[1] Granat, M., Ferguson, A., Andrews, B. \& Delargy, M., The role of functional electrical stimulation in the rehabilitation of patients with incomplete spinal cord injury - observed benefits during gait studies. Paraplegia, 31, 1993.

[2] Petrofsky, J. \& Phillips, C., Closed loop control for restoration of movement in paralyzed muscle. J Orth, 9, pp. 13-19, 1984.

[3] Phillips, C., Koubek, R. \& Hendershot, D., Walking while using a sensory tactile feedback system: potential use with a functional electrical stimulation orthosis. J Biomed Eng, 13(3), pp. 91-96, 1991.

[4] Mulder, T. \& Hochstenbach, J., Motor Control and Learning: Implications for Neurological Rehabilitation. Hillsdale: Eribaum Publ.: Handbook of Neurological Rehabilitation, in press.

[5] Erzin, R., Bajd, T., Kralj, A., Šavrin, R. \& Benko, H., Influence of sensory biofeedback on FES assisted walking. Electrotechnical Review, 63, 1996.

[6] Bajd, T., Kralj, A., Štefančič, M. \& Lavrač, N., Use of functional electrical stimulation in the lower extremities of incomplete spinal cord injured patients. Artif Organs, 23(5), pp. 403-409, 1999.

[7] Cikajlo, I. \& Bajd, T., Swing phase estimation in paralyzed persons walking. Tech Health \& Care, in press.

[8] Bajd, T., Cikajlo, I., Šavrin, R., Erzin, R. \& Gider, F., FES rehabilitative systems for re-education of walking in incomplete spinal cord injured persons. Neuromodulation, 3(3), pp. 167-174, 2000.

[9] Cikajlo, I. \& Bajd, T., FES gait re-education : the swing phase estimation. 6th Annual Conference of the International Functional Electrical Stimulation Society, eds. T. Karčnik, P. Veltink \& R. Jaeger, Ljubljana: Ministry of Education, Science and Sport of the Republic of Slovenia, pp. 49-51, 2002. 